\title{
Caveolar dysfunction and lipodystrophies
}

\author{
Nivedita Patni ${ }^{1}$, Robert A Hegele $\mathbb{D}^{2}$ and Abhimanyu Garg ${ }^{3}$ \\ ${ }^{1}$ Division of Pediatric Endocrinology, Department of Pediatrics, UT Southwestern Medical Center, Dallas, Texas, USA, \\ ${ }^{2}$ Department of Medicine and Robarts Research Institute, Western University, London, Ontario, Canada, and \\ ${ }^{3}$ Division of Nutrition and Metabolic Diseases, Department of Internal Medicine and the Center for Human Nutrition, \\ UT Southwestern Medical Center, Dallas, Texas, USA
}

Congenital generalized lipodystrophy (CGL) is a rare, heterogeneous, autosomal recessive disorder characterized by the near-total absence of body fat with increased muscularity noticed at birth or in early infancy. Four distinct genetic subtypes of CGL have been reported to date. Types 1 and 2 are caused by biallelic variants in the 1-acylglycerol-3-phosphate-O-acyltransferase 2 (AGPAT2) and Berardinelli-Seip congenital lipodystrophy 2 (BSCL2) genes, respectively and are the most common subtypes (1). Types 3 and 4 are extremely rare and are caused by biallelic variants in the caveolin 1 (CAV1) (2), and caveolaeassociated protein-1 (CAVIN1; also known as polymerase I and transcript release factor (PTRF)]) genes (3), respectively. Patients with all CGL subtypes are predisposed to metabolic complications of insulin resistance, such as diabetes mellitus, hypertriglyceridemia, and hepatic steatosis; however, each subtype presents with some unique clinical features.

Type 3 CGL was initially reported in the landmark study of a single 20-year-old Brazilian female belonging to a consanguineous family with a homozygous p.(Glu38*) CAV1 variant (2). She had generalized lipodystrophy, short stature, functional megaesophagus, severe hypertriglyceridemia, primary amenorrhea, chronic diarrhea, hepatic steatosis, and splenomegaly, and hypocalcemia presumed to be secondary to vitamin D resistance (2). She also had early onset acanthosis nigricans and hirsutism and developed diabetes at 13 years of age. She had absence of metabolically active adipose tissue, but mechanical adipose tissue and bone marrow fat were well-preserved (2). However, it was not clear which phenotypic features of this patient could be specifically attributed to the homozygous CAV1 variant; additional patients were required to prove the diseasegene association.
Correspondence

should be addressed

to A Garg

Email

abhimanyu.garg@

utsouthwestern.edu
A recent artice published in this journal by Karhan et al. (4) has provided further compelling evidence on the association of CGL3 phenotype with a novel homozygous CAV1 p.(His79Glnfs*3) variant by reporting the detailed phenotype of four additional patients, three females (age range, 8 months to 15 years) and one male (age 18 years), from a large Turkish kindred. All patients developed lipodystrophy in early infancy, had short stature, and failure to thrive, despite a voracious appetite. All three females had severe metabolic abnormalities including hypertriglyceridemia, low levels of high-density lipoprotein (HDL) cholesterol, and fasting hyperinsulinemia at a young age. The 15-year-old female had acanthosis nigricans, hirsutism, polycystic ovaries, and hepatic steatosis. The 18-year-old male had normal serum triglycerides and mildly decreased HDL-cholesterol and insulin resistance. Both older patients, but not the 10-year-old affected female, had dysphagia and megaesophagus due to achalasia requiring peroral endoscopic myotomy. Kim et al. (2) also reported functional megaesophagus, which was corrected at 15 years of age in their patient; however, further details were not provided. Karhan et al. (4) have suggested dysregulation of calcium homeostasis and excitation-contraction coupling in smooth muscle due to caveolae deficiency as potential cause of achalasia.

Kim et al. (2) had described hypocalcemia and renal calcium wasting, along with radiographic findings suggestive of osteopenia in their patient, requiring vitamin D supplementation. However, all four patients reported by Karhan et al. (4) had normal serum and urinary calcium levels. The two older patients, a 15-year-old female and 18-year-old male, had reduced bone mineral density suggestive of osteopenia (lumbar z score -2.78 and -3.85 , respectively). Karhan et al. (4) also reported a bilateral loss of ellipsoid line and atrophy of the retinal pigment

Published by Bioscientifica Ltd. 
epithelium, suggestive of atypical retinitis pigmentosa in the 18-year-old male. Some recent studies have shown that $C A V 1$ is expressed in several retinal cell types and can modulate neuroprotective signaling (5). Interestingly, bilateral retinitis pigmentosa has been previously reported in two patients with a heterozygous CAV1 p. (Lys135Argfs ${ }^{*} 4$ ) variant, along with partial lipodystrophy and hypertriglyceridemia (6).

Karhan et al. (4) also reported six siblings with normal genotype and two siblings and seven parents with heterozygous CAV1 p.(His79Glnfs*3) variant in the index kindred. They reported no lipodystrophy or metabolic derangements in the heterozygous carriers, although detailed anthropometric, metabolic, and bone density data were not available. For example, comparing the height and bone density of the affected homozygotes, heterozygous parents and siblings, andsiblings with normal genotype would have confirmed their observation of short stature and osteopenia in the affected subjects. Furthermore, a whole exome or genome study of the family would have excluded other causal variants and their association, if any, with the phenotypes such as atypical retinitis pigmentosa.

Another intriguing fact is that while the heterozygous parents and siblings from the two CGL3 pedigrees harboring the null CAV1 variants (i.e. p.(Glu38*) and p.(His79Glnfs*3)) have a normal phenotype consistent with autosomal recessive inheritance, previously other heterozygous variants c.-88delC, p.(Lys135Argfs*4), p. $($ Gln142*), p.(Phe160*), p.(Pro158Hisfs*23), and p.(Leu159Serfs*22) have been reported in association with pulmonary hypertension, and progressive right ventricular failure (7), partial lipodystrophy (6), and neonatal-onset lipodystrophy and progeroid syndrome (8). How these different heterozygous CAV1 variants, even though they are all apparently null, cause phenotypes ranging from normal to severe syndromes is intriguing. There is a possibility that some of the heterozygous variants lead to a phenotype due to dominant-negative mechanisms, whereas the p.(Glu38*) and p.(His79Glnfs*3) variants do not because of true haploinsufficiency. The phenotypic variability among some of the heterozygotes could also be due to digenic interactions or polygenic influences.

The molecular basis of CGL3 and CGL4 implicates caveolar-dependent mechanisms in lipodystrophy. Caveolae are flask-shaped invaginations on the plasma membranes that maintain the integrity and function of the lipid droplets. They are mainly composed of lipids such as sphingolipids and cholesterol and proteins, such as caveolins and CAVINs. In addition, pacsin/syndapin proteins, Eps15 homology domain proteins 1, 2, and 4 , and receptor tyrosine kinase-like orphan receptor 1 (ROR1) have been recently reported to play a role in caveolae formation (9). CAV1 and CAV2 are expressed ubiquitously except in striated muscle and cardiac muscle cells, and CAV1 is highly expressed in the adipocytes, endothelial cells, and fibroblasts; however, CAV3 is muscle-specific (9).

Most interesting among these caveolar proteins is CAVIN1, as biallelic variants in this protein cause CGL4, which has many similar features to CGL3 (see Table 1). CAVIN1 anchors and stabilizes CAV1 to the cytoskeleton in adipocytes, and CAVIN1 deficiency causes reductions of caveolae with a barely detectable expression of CAV1 and CAV2 and reduced expression of CAV3 in human skeletal muscle (3). Similarly, skin fibroblasts of the patients with homozygous CAV1 p.(Glu38*) and p.(His79Glnfs*3) variants did not exhibit any detectable protein expression of CAV1 and strongly decreased expression of CAV2 and CAVIN1 (4). Therefore, the observed overlapping phenotypic features between CGL3 and CGL4 are not surprising. In fact, unique clinical features of CGL3 and CGL4 can be exploited to further our understanding of the roles of CAV1 and CAVIN1 in caveolae formation in various human tissues.

Patients with CGL4 have congenital pyloric stenosis; however, one patient has been described to have achalasia, diagnosed after she presented with dysphagia at 14 years of age (10). The autopsy report of a 14-year-old male with CGL4 showed thickened muscularis mucosa in the esophagus and gastro-duodenal junction, and prominent/large nerves in the esophagus, consistent with clinical features of esophageal dysmotility, infantile hypertrophic pyloric stenosis, and ileus in the patient (11). Pathophysiology of achalasia has been attributed to the functional loss of myenteric plexus ganglion cells in the distal esophagus and lower esophageal sphincter; however, the pathophysiology in CGL3 and CGL4 is unclear. Patients with type 4 CGL have congenital myopathy with high serum creatine kinase levels, 'muscle mounding', osteopenia, joint stiffness, atlantoaxial instability, and serious arrhythmias such as catecholaminergic polymorphic ventricular tachycardia, and sudden death (1). Similar to CGL3, metabolically active adipose tissue is absent in patients with CGL4, but mechanical adipose tissue and bone marrow fat are preserved. Table 1 describes unique clinical features for the CGL 3 and 4 subtypes.

Interestingly, knockout mouse models of Cav1 and Cavin1 also have distinct phenotypes. Cav1 $1^{-/-}$mice have 
Table 1 Clinical features of congenital generalized lipodystrophy type 3 and type 4 .

\begin{tabular}{|c|c|}
\hline & Type 3 CGL \\
\hline Growth & $\begin{array}{l}\text { Short stature } \\
\text { Failure to thrive in infancy } \\
\text { Generalized lipodystrophy }\end{array}$ \\
\hline Ophthalmologic & Retinitis pigmentosa* \\
\hline Cardiovascular & None \\
\hline Gastrointestinal & $\begin{array}{l}\text { Achalasia/megaesophagus } \\
\text { Hepatic steatosis }\end{array}$ \\
\hline Musculoskeletal & Osteopenia \\
\hline Genitourinary & $\begin{array}{l}\text { Polycystic ovaries* } \\
\text { Amenorrhea }\end{array}$ \\
\hline Neuro-cognitive & None \\
\hline
\end{tabular}

Type 4 CGL

Generalized lipodystrophy

None

Catecholaminergic polymorphic ventricular tachycardia Prolonged QT interval

Sudden death

Congenital pyloric stenosis

Hepatic steatosis

Achalasia*

Myopathy

Muscle mounding

Joint stiffness

Atlanto-axial instability

Osteopenia

Azoospermia\#

None

*Described in a single patient; "Described in autopsy (11).

hyperphagia but resistance to high-fat diet-induced obesity and have severely elevated post-prandial triglycerides and free-fatty acid levels; however, serum insulin, glucose, and cholesterol levels are entirely normal (12). They have a systemic decompensation in lipid accumulation with increasing age, resulting in dramatically smaller fat pads, reduced adipocyte cell diameter, and a poorly differentiated/ hypercellular white adipose parenchyma. Cavin $1^{-/-}$mice, on the other hand, have high circulating triglyceride levels, reduced adipose tissue mass, hyperinsulinemia, and glucose intolerance, consistent with lipodystrophic phenotype. They lack morphologically detectable caveolae, have impaired triacylglycerol uptake and storage by adipocytes, and have hypertrophy of cardiomyocytes accompanied by progressive interstitial/perivascular fibrosis $(3,13)$. The relationship of these mouse models to the corresponding human deficiencies should be followed up.

In conclusion, the report by Karhan et al. (4) expands and provides further clarification of the CGL3 phenotype associated with homozygous CAV1 null variants. The findings solidify the central role of caveolae in human lipodystrophy syndromes and are hypotheses-generating with respect to explaining the underlying phenotypic heterogeneity. Additional reports of this rare disorder in the future will be required to understand its natural history.

\section{Declaration of interest}

The authors declare that there is no conflict of interest that could be perceived as prejudicing the impartiality of this commentary.

\section{Funding}

A G and N P are supported by National Institutes of Health grant R01DK105448 and A G is also supported by the Southwestern Medical Foundation. R A H is supported by the Jacob J. Wolfe Distinguished Medical Research Chair, the Edith Schulich Vinet Research Chair, and the Martha G. Blackburn Chair in Cardiovascular Research. R A H holds operating grants from the Canadian Institutes of Health Research (Foundation award), the Heart and Stroke Foundation of Ontario (G-21-0031455), and the Academic Medical Association of Southwestern Ontario (INN21-011).

\section{References}

1 Patni N \& Garg A. Congenital generalized lipodystrophies--new insights into metabolic dysfunction. Nature Reviews: Endocrinology 201511 522-534. (https://doi.org/10.1038/nrendo.2015.123)

2 Kim CA, Delepine M, Boutet E, El Mourabit H, Le Lay S, Meier M, Nemani M, Bridel E, Leite CC, Bertola DR et al. Association of a homozygous nonsense caveolin-1 mutation with BerardinelliSeip congenital lipodystrophy. Journal of Clinical Endocrinology and Metabolism 200893 1129-1134. (https://doi.org/10.1210/jc.2007-1328)

3 Hayashi YK, Matsuda C, Ogawa M, Goto K, Tominaga K, Mitsuhashi S, Park YE, Nonaka I, Hino-Fukuyo N, Haginoya K et al. Human PTRF mutations cause secondary deficiency of caveolins resulting in muscular dystrophy with generalized lipodystrophy. Journal of Clinical Investigation 2009119 2623-2633. (https://doi.org/10.1172/JCI38660)

4 Karhan AN, Zammouri J, Auclair M, Capel E, Apaydin FD, Ates F, Verpont MC, Magre J, Feve B, Lascols O et al. Biallelic CAV1 null variants induce congenital generalized lipodystrophy with achalasia. European Journal of Endocrinology 2021185 841-854. (https://doi. org/10.1530/EJE-21-0915)

5 Reagan A, Gu X, Hauck SM, Ash JD, Cao G, Thompson TC \& Elliott MH. Retinal Caveolin-1 modulates neuroprotective signaling. Advances in Experimental Medicine and Biology 2016854 411-418. (https://doi.org/10.1007/978-3-319-17121-0_54)

6 Cao H, Alston L, Ruschman J \& Hegele RA. Heterozygous CAV1 frameshift mutations (MIM 601047) in patients with atypical partial lipodystrophy and hypertriglyceridemia. Lipids in Health and Disease 20087 3. (https://doi.org/10.1186/1476-511X-7-3)

7 Austin ED, Ma L, LeDuc C, Berman Rosenzweig E, Borczuk A, Phillips JA, 3rd, Palomero T, Sumazin P, Kim HR, Talati MH et al. 
Whole exome sequencing to identify a novel gene (caveolin-1) associated with human pulmonary arterial hypertension. Circulation. Cardiovascular Genetics 20125 336-343. (https://doi.org/10.1161/ CIRCGENETICS.111.961888)

8 Garg A, Kircher M, Del Campo M, Amato RS, Agarwal AK \& University of Washington Center for Mendelian Genomics. Whole exome sequencing identifies de novo heterozygous CAV1 mutations associated with a novel neonatal onset lipodystrophy syndrome. American Journal of Medical Genetics: Part A 2015 167A 1796-1806. (https://doi.org/10.1002/ajmg.a.37115)

9 Parton RG, McMahon KA \& Wu Y. Caveolae: formation, dynamics, and function. Current Opinion in Cell Biology 202065 8-16. (https:// doi.org/10.1016/j.ceb.2020.02.001)

10 van der Pol RJ, Benninga MA, Magre J, Van Maldergem L, Rotteveel J, van der Knaap MS \& de Meij TG. Berardinelli-Seip syndrome and achalasia: a shared pathomechanism? European Journal of Pediatrics 2015174 975-980. (https://doi.org/10.1007/s00431015-2556-y)

11 Patni N, Vuitch F \& Garg A. Postmortem findings in a young man with congenital generalized lipodystrophy, type 4 due to CAVIN1 mutations. Journal of Clinical Endocrinology and Metabolism 2019104 957-960. (https://doi.org/10.1210/jc.2018-01331)

12 Razani B \& Lisanti MP. Caveolin-deficient mice: insights into caveolar function human disease. Journal of Clinical Investigation 2001108 1553-1561. (https://doi.org/10.1172/JCI14611)

13 Taniguchi T, Maruyama N, Ogata T, Kasahara T, Nakanishi N, Miyagawa K, Naito D, Hamaoka T, Nishi M, Matoba S et al. PTRF/ Cavin-1 deficiency causes cardiac dysfunction accompanied by cardiomyocyte hypertrophy and cardiac fibrosis. PLOS ONE 201611 e0162513. (https://doi.org/10.1371/journal.pone.0162513) 